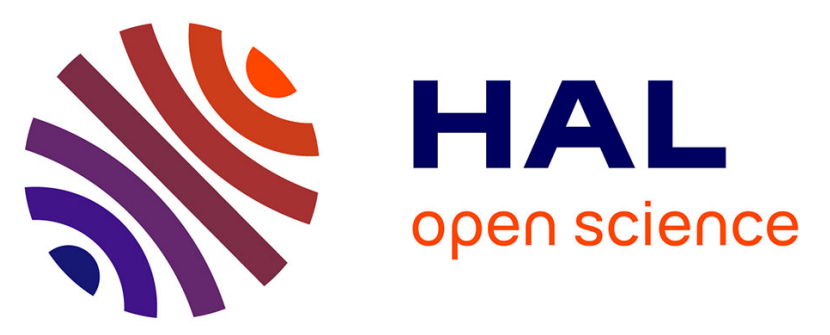

\title{
Atomic Scale Modelling of Two-Dimensional Molecular Self-Assembly on a Passivated Si Surface
}

Guillaume Copie, Younes Makoudi, Christophe Krzeminski, Frederic

Cherioux, Frank Palmino, Simon Lamare, B. Grandidier, Fabrizio Cleri

\section{To cite this version:}

Guillaume Copie, Younes Makoudi, Christophe Krzeminski, Frederic Cherioux, Frank Palmino, et al. Atomic Scale Modelling of Two-Dimensional Molecular Self-Assembly on a Passivated Si Surface. Journal of Physical Chemistry C, 2014, 118 (24), pp.12817-12825. 10.1021/jp501955v . hal-01015467

\section{HAL Id: hal-01015467 \\ https://hal.science/hal-01015467}

Submitted on 26 Jun 2014

HAL is a multi-disciplinary open access archive for the deposit and dissemination of scientific research documents, whether they are published or not. The documents may come from teaching and research institutions in France or abroad, or from public or private research centers.
L'archive ouverte pluridisciplinaire HAL, est destinée au dépôt et à la diffusion de documents scientifiques de niveau recherche, publiés ou non, émanant des établissements d'enseignement et de recherche français ou étrangers, des laboratoires publics ou privés. 


\section{Atomic Scale Modelling of Two-Dimensional}

\section{Molecular Self-Assembly on a Passivated Si Surface}

G. Copie ${ }^{\dagger}$ Y. Makoudi, ${ }^{\ddagger}$ C. Krzeminski, ${ }^{\mathbb{I}}$ F. Chérioux,$\ddagger$ F. Palmino, ${ }^{\ddagger}$ S. Lamare,${ }^{\ddagger}$ B. Grandidier, ${ }^{\mathbb{I}}$ and F. Cleri*, $\dagger$

Institut d'Electronique, Microélectronique et Nanotechnologie (IEMN), CNRS UMR 8520,

Université de Lille 1 Sciences et Technologies, Avenue Poincaré, 59652 Villeneuve d'Ascq,

France, Institut FEMTO-ST, Université de Franche-Comté, CNRS, ENSMM, 32 avenue de

l'Observatoire, 25044 Besançon cedex, France, and Institut d'Electronique, Microélectronique et

Nanotechnologie (IEMN), CNRS UMR 8520, Département ISEN, Avenue Poincaré, 59652

Villeneuve d'Ascq, France

E-mail: fabrizio.cleri@univ-lille1.fr

\footnotetext{
${ }^{*}$ To whom correspondence should be addressed

†'Institut d'Electronique, Microélectronique et Nanotechnologie (IEMN), CNRS UMR 8520, Université de Lille 1 Sciences et Technologies, Avenue Poincaré, 59652 Villeneuve d'Ascq, France

¥Institut FEMTO-ST, Université de Franche-Comté, CNRS, ENSMM, 32 avenue de l'Observatoire, 25044 Besançon cedex, France

IIInstitut d'Electronique, Microélectronique et Nanotechnologie (IEMN), CNRS UMR 8520, Département ISEN, Avenue Poincaré, 59652 Villeneuve d'Ascq, France
} 


\begin{abstract}
The self-assembly of two-dimensional (2D) molecular structures on a solid surface relies on the subtle balance between non covalent intermolecular and molecule-surface forces. The energetics of 2D molecular lattices forming different patterns on a passivated semiconductor surface are here investigated by a combination of atomistic simulation methods. Densityfunctional theory provides structure and charges of the molecules, while metadynamics with empirical forces provides a best guess for the lowest-energy adsorption sites of single molecules and dimers. Subsequently, molecular dynamics simulations of extended molecular assemblies with empirical forces yield the most favorable lattice structures at finite temperature and pressure.
\end{abstract}

Keywords: Scanning electron microscopy; molecular dynamics; molecular dispersion forces; physisorption; polyphenil organic molecules 


\section{Introduction}

The need for a hierarchical, spontaneous assembly of nanoscale building blocks is very strong in the nanoscience community, as a potential solution to the problems inherent with the manipulation and positioning of such small scale objects with atomic-scale precision. ${ }^{1}$ Two-dimensional selfassembled lattices of organic molecules linked by non-covalent forces (mainly Van der Waals, electrostatic, hydrogen-bond) exhibit a wealth of fascinating physical phenomena, which suggest many interesting technological applications. ${ }^{2-4}$ However, the controlled construction of an adsorbed (supra)molecular lattice suffers from a lack of knowledge regarding the role of dispersion forces, compared to the relative ease of manipulating other types of intermolecular forces in supramolecular chemistry. ${ }^{5-7}$ As a result, it is always difficult to realistically predict the detailed molecular architecture of the final self-assembled lattice. This is particularly true for the

increasing number of supramolecular structures synthesized on non-metallic surfaces, ${ }^{8-14}$ where the molecule-surface interaction is much less investigated, compared to the case of molecules on metal surfaces. ${ }^{5-7}$ Therefore, the development of computer simulation methods, to study the competition between such poorly known forces, and to ultimately predict the supramolecular architectures on the surface, may reveal an essential step in the design of self-assembled molecular structures, moreover representing a savings of experimental time in the empirical trial-and-error search for candidate configurations.

Due to the high number of competing configurations which may be adopted by the molecules on a surface, it is practically impossible to obtain the minimum energy configurations from a strictly theoretical approach, except in extremely simple cases of very high symmetry. In practice an initial molecular structure must be guessed on the basis of experimental observations. However, multi-scale atomistic simulations can offer an alternative approach: firstly, density-functional theory (DFT) and metadynamics (MtD) can provide a best guess for the lowest-energy adsorption sites of either a single molecule, or small molecular clusters as a starting point. Subsequently, molecular dynamics (MD) for a large assembly of molecules can follow the phase evolution at finite temperature and pressure, and identify candidate supramolecular lattices. Indeed, MD with either empirical 
or ab-initio forces is a powerful computer simulation method to probe the structure of molecular assemblies, and to extract the relative importance of the various molecular (non covalent) forces involved in assembling the structure. MD has been successfully applied, for example, to the study of molecular monolayers adsorbed on graphite. ${ }^{15-18}$ In a general perspective, a final simulation step by large-scale kinetic Monte-Carlo (KMC) can use all the above information to study the phase evolution over macroscopic time and length scales, under varying thermodynamical constraints. ${ }^{19}$ The as-obtained theoretical structures should be directly comparable to experimental results.

In this work we use such a multi-scale strategy (only excluding the last KMC step, which would not add further insight for this particular case) to study the self-assembly of molecular monolayers at surfaces, in comparison with experimentally synthesized structures. We choose to work with a semiconductor surface, instead of the much better studied metal surfaces. ${ }^{5,20}$ Indeed, semiconductor surfaces are not expected to behave as a catalyst surfaces, ${ }^{21}$ since no d-band exist close to the Fermi level for conventional semiconductors. Substrate-mediated interactions through surface states electrons $^{22}$ are also precluded because surface states are highly reactive on semiconductor surfaces, what leads to the formation of covalent bonds. Finally, molecular redistribution through screening effects are expected to be much weaker. ${ }^{23}$ In addition to the need for deeper insight into the chemistry of supramolecular arrangements on semiconductor surfaces, interesting prospects are foreseen based on recent works that used two-dimensional molecular assemblies to design very fine sacrificial masks on conventional semiconductor materials ${ }^{24}$ or create nanoscale electroactive template for biosensing applications. ${ }^{25}$ We take advantage of the relatively inert character of the boron-doped $\mathrm{Si}: \mathrm{B}(111)(\sqrt{3} \times \sqrt{3}) \mathrm{R} 30^{\circ}$ surface, indicated as $\mathrm{Si}: \mathrm{B}(111)$, to prevent the covalent bonding of molecules to the surface. ${ }^{8,12-14,19}$ The particular structure and geometry of this surface are of great interest to study the competition between intermolecular and molecule-surface interactions in the construction of self-assembled, ordered molecular lattices. ${ }^{19}$ We consider a family of molecules with a prototypical structure whose backbone consists of phenyl groups, linked in a way that ensures a ternary symmetry. Indeed, such molecules with a 1,3,5-tri(phenyl)benzene backbone have already been shown to interact very weakly with the Si:B (111) surface at room 
temperature. ${ }^{11}$ Here, different molecule lengths as well as different end-groups were considered, in order to characterize the relative strength of intermolecular vs. molecule-surface forces, as well as to elucidate the role of dispersion forces, compared to specific head-group interactions. Firstly, we use DFT to determine the initial structure and charge distribution of our molecules. Then, MtD with empirical force fields is performed for single, isolated molecules on the surface, for which the role of the molecular size and termination in the configuration adsorbed at different surface sites is highlighted; on the other hand, when applied to molecular dimers, MtD allows to determine the conformation that maximizes intermolecular interactions. Subsequenty, based on the as-identified elementary building blocks, we define several two-dimensional supramolecular lattices, on which large-scale MD simulations at finite temperature are performed. Finally, we compare the relative dynamical stability of the various lattice arrangements obtained from MD, for each type of molecule, against experimental images of molecular structures synthesized in our laboratory. We demonstrate that the combined simulations do successfully predict in all cases the most probable molecular architecture experimentally observed, thereby adding valuable insight to the knowledge of the subtle balance existing between the various molecular forces involved in the self-assembly process. 


\section{Materials and Methods}

\section{Computer simulations}

We arrange 1,3,5-tris(4'-X-Y-phenyl)benzene molecules ( $\mathrm{X}=\mathrm{H}$ or $\mathrm{Br}$, Y=mono or bi, see Figure 1) on a periodically-repeated $\mathrm{Si}: \mathrm{B}(111)$ surface. The molecular backbone is formed by three identical arms, each made of one or two phenyl rings, attached to a central phenyl at the 1, 3 and 5 carbons, and terminated by a $\mathrm{H}$ or a $\mathrm{Br}$ atom. The average length of each arm is 10.6 or $11.5 \AA$, respectively, from the center of the central phenyl group. In principle, all the ground state conformations of the molecules should be characterized by a $C_{3 v}$ planar symmetry. However, when interacting with the surface and other molecules, the phenyl arms can rotate and slightly bend relative to each other, to maximize the interaction via Van der Waals forces and the formation of hydrogen bonds, thus partially disrupting the perfect symmetry. For the subsequent simulations, effective charges for the isolated molecules in void are assigned by the Merz-Singh-Kollman (MK) definition of the molecular electrostatic potential, as implemented in Gaussian $03^{26}$ single-point calculations with the B3LYP/6-311g functional. For both molecules, we find that $\mathrm{H}$ charges are always comprised between neutral and $0.2 e$; $\mathrm{C}-\mathrm{C}$ bound atoms are between neutral and $+0.3 e$. $\mathrm{C}$ bound to $\mathrm{H}$ are between -0.1 and $-0.3 e$, except for $\mathrm{C}$ in the center of the THBB molecule which are at $+0.2 e$, and $\mathrm{C}$ neighboring the $\mathrm{C}-\mathrm{Br}$ function, which are at about $+0.1 e . \mathrm{Br}$ atoms are at $-0.05 e$.

The Si:B(111) surface doped with boron is a well-studied case (see ${ }^{19}$ and refs. therein), the B dopant atoms going subsurface and pushing a Si atom above the surface by $2.18 \AA$, thereby inducing a $\sqrt{3} \times \sqrt{3}$ reconstruction, with a rhombic unit cell with side $a=6.65 \AA$ and surface 22.1 $\AA^{2} .{ }^{19}$ According to our experimental indications, which suggest an inert, unpolarised surface, the Si:B (111) surface was assigned a neutral charge state at every site.

MD simulations were performed with the DLPOLY4 code, ${ }^{27}$ and DLPOLY2 code with the plugin PLUMED 28,29 for metadynamics. We choose to freeze the Si:B (111) surface in order to simplify the problem. The $\mathrm{Si}$ atomic lattice is built by minimizing the diamond structure con-

figuration with the Stillinger-Weber empirical potential ${ }^{30}$ and the extension to Si-B by Rasband 
et at. ${ }^{31}$ which gives an equilibrium lattice parameter $a_{0}=0.543 \mathrm{~nm}$. To describe the atom-atom interactions in molecules we used the MM3 force field, ${ }^{32,33}$ which includes bonding (stretching, bending, torsional, inversion) and non-bonding (Van der Waals, Coulomb and eventual H-bonding) force terms. Atomic trajectories in $\mathrm{MD}$ and $\mathrm{MtD}$ at finite temperature $T \leq 10 \mathrm{~K}$ are calculated by the velocity Verlet integrator, with a time step of $1 \mathrm{fs}$. Long-range electrostatic forces are evaluated using Ewald sum.

Short-range Van der Waals (VdW) interactions in the MM3 force field are described by a Buckingham-type two-body potential with a cutoff of $9 \AA$ :

$$
U\left(r_{i j}\right)=A \exp \left(-r_{i j} / \rho\right)-C r_{i j}^{-6}
$$

with $A, \rho$ and $C$ parameters depending on the atom species of $i$ and $j$; and $r_{i j}$ the distance between the two atoms $i$ and $j$.

Notably, such a spherically-symmetric potential evolves into a $R^{-6}$ dependence, once summed over all the atoms belonging to two objects (molecule or surface) at an average distance $R$. From the theory of VdW forces and the related Hamaker coefficients, however, it is known that this is not the best way to describe the interaction between a "small" object and a flat surface, ${ }^{34}$ which should rather evolve into a $R^{-3}$ dependence at large distances. To address such a problem, we adopted a new potential model for the molecule-surface interaction, which was recently proposed on the basis of ab-initio TD-DFT calculations: ${ }^{35}$

$$
U(z)=A \exp \left(-\left(z-z_{0}\right) / b\right)-C\left(z-z_{0}\right)^{-3}
$$

In the equation above, which represents the best fit to the ab-initio data for a large number of hydrocarbon-Si configurations, $A, b$ and $C$ are fitting parameters depending on both the atomic species and the surface (see Table 2 in Ref. 35); $z_{0}$ a constant representing the effective surface quota; and $z$ the Cartesian coordinate of the atom above the surface. The origin of the $z$-axis is taken at the top of the surface, and for the case of perfect Si surface, $z_{0}$ is equal to $d / 2$ with $d$ the 
interplanar distance. We retained such a value also for our surface since, despite the high level of B doping, the surface remains semiconductor. (The B atoms are located below the first surface layer, see description above, and our experiments do not show any traces of surface polarisation.) This potential function describes the surface as a continuum, and the molecule as an assembly of atoms interacting with this surface according to their $\left(z-z_{0}\right)$ relative position. With this choice, the interaction energy correctly evolves into $R^{-3}$ for a molecule at a distance $R=|\mathbf{R} \cdot \hat{\mathbf{z}}|$ above the surface.

\section{Molecule and substrate preparation}

1,3,5-tri(phenyl)benzene (THB) and 1,3,5-tri(4'-bromophenyl)benzene (TBB) were purchased from Sigma-Aldrich Inc., purified by column chromatography on silica gel, and then sublimated. 1,3,5tri(4,4'-biphenyl)-benzene (THBB) and 1,3,5-tri(4'-bromo-4,4'-biphenyl)-benzene (TBBB) were synthetized by cyclotrimerization of 4-phenyl-acetophenone and of 4'-bromophenyl-4-acetophenone, respectively in trifluoromethane sulfonic acid. ${ }^{36-38}$

Synthesis of 1,3,5-tri(4,4'-biphenyl)benzene (THBB): 4-phenyl-acetophenone (3.14g, 16 mmol) and trifluoromethane sulfonic acid $(1 \mathrm{~mL})$ were dissolved in $40 \mathrm{~mL}$ of degassed toluene at room temperature. Then, the mixture was heated at reflux for $14 \mathrm{~h}$. The mixture was subsequently cooled to room temperature, and $100 \mathrm{ml}$ of toluene was added. The organic solution was washed two times with $100 \mathrm{~mL}$ of aqueous $\mathrm{K}_{2} \mathrm{CO}_{3}(0.5 \mathrm{M})$ and two times with $100 \mathrm{~mL}$ of water. The solvent was removed under reduced pressure. The resulting brown solid was washed two times with 40 $\mathrm{mL}$ of diethylether. The pale yellow solid was recrystallized in a toluene/ethanol 1:1 solution to give a pure white solid. All molecules have been subsequently purified by column chromatography on silica gel and sublimated. ${ }^{1} \mathrm{H}$ NMR $(300 \mathrm{MHz}$, Chloroform- $d) \delta 7.90(\mathrm{~s}, 3 \mathrm{H}), 7.82(\mathrm{~d}, J=8.2$ Hz, 6H), $7.74(\mathrm{~d}, J=8.2 \mathrm{~Hz}, 6 \mathrm{H}), 7.68(\mathrm{~d}, J=7.1 \mathrm{~Hz}, 6 \mathrm{H}), 7.49(\mathrm{t}, J=7.3 \mathrm{~Hz}, 6 \mathrm{H}), 7.43-7.32(\mathrm{~m}$, $3 \mathrm{H})$.

High quality boron-doped $\operatorname{Si}(111)(\sqrt{3} \times \sqrt{3}) \mathrm{R} 30^{\circ}$ surfaces were obtained by annealing heavily boron-doped $\mathrm{Si}(111)$ crystals at a temperature higher than $900^{\circ} \mathrm{C}$ for a few hours in ultra high 
vacuum. ${ }^{39}$ Deposition of THBB and TBBB molecules was performed at 202 and $250^{\circ} \mathrm{C}$, respectively, onto the Si:B (111) surface maintained at room temperature with a base pressure less than $2 \times 10^{-10}$ mbar. The self-assembled monolayers were subsequently imaged with scanning tunneling microscopy in constant-current mode, with a sample bias range of 3.9 to $2.3 \mathrm{~V}$ for THBB, and a positive range of 2.3 to $1.6 \mathrm{~V}$ and negative range of -2.4 to $-1.6 \mathrm{~V}$ for TBBB. The tunneling current was $10 \mathrm{pA}$.

It is worth noting that the adsorption of THBB and TBBB molecules on Si:B (111) is described for the first time. These molecules possess an additional phenyl ring on each arm relatively to THB and TBB molecules. This difference is expected to modify the molecule-surface and molecule-molecule interactions, leading to more stable arrangements in particular for the hydrogen terminated complex. ${ }^{11}$ 


\section{Results and Discussion}

\section{Metadynamics of single molecules and dimers}

Firstly we used MtD to identify the lowest-energy adsorption sites for each isolated molecule on the $\mathrm{Si}: \mathrm{B}$ (111) surface. We started by depositing a single molecule at a random site on the surface. The system was then relaxed by the steepest descent method. Subsequently, we began the proper $\mathrm{MtD}$ simulation. We used as collective variables (CV) the $x$ and $y$ coordinates of the molecular center of mass. ${ }^{40}$ The Gaussian functions necessary in MtD to fill the true potential well, had a width of $0.35 \AA$, height of $0.4 \mathrm{kcal} / \mathrm{mol}$, and were introduced at intervals of $1 \mathrm{ps}$, during a $2 \mathrm{~ns}$ simulation at $T=1 \mathrm{~K}$. In order to limit the size of the $\mathrm{CV}$ space to be explored, and because of the symmetry of the surface, we restrained the molecule displacement within a $23.1 \times 13.4 \AA^{2}$ area, centered on a unit cell of the Si:B (111) surface. For each molecule, we repeated the MtD simulation several times, with different initial conditions, and then averaged the results to produce a map of the free energy surface, as shown in Figure 2.

For the smaller THB and TBB molecules (upper row of Fig. 2), it appears that the "hollow" site, located among three Si adatoms (black dots) has the lowest free energy; the adsorption free energy is larger for the TBB $(\approx-5.5 \mathrm{kcal} / \mathrm{mol})$. It can be noted that the free energy map around the minimum has a triangular symmetry. For the TBB, a secondary minimum also exists, on the "top" site right above the $\mathrm{Si}$ adatom, albeit at a smaller adsorption free energy $(\approx-2 \mathrm{kcal} / \mathrm{mol})$.

For the larger molecules (lower row of Fig. 2), an opposite behavior is observed. The TBBB displays a distinct free-energy minimum $(\approx-5 \mathrm{kcal} / \mathrm{mol})$ at the "top" site, interestingly with a very symmetric, circular shape, indicating a greater freedom in the in-plane orientation. As to the "hollow" site, it corresponds to a secondary minimum, with a smaller adsorption free energy $(\approx-3 \mathrm{kcal} / \mathrm{mol})$. The THBB shows the same two minima at the "hollow" and "top" site, however with nearly the same value of free energy $(\approx-4 \mathrm{kcal} / \mathrm{mol})$. It is worth noting that for all the four molecules, the regions connecting two surface adatoms have a nearly zero adsorption free energy. Therefore, these "bridge" elongated sites would represent energy barriers for the diffusion 
of the molecules on the Si:B (111) surface. In summary, in this first set of MtD simulations we highlighted the role of $\mathrm{VdW}$ forces between a single molecule and the surface, since neither electrostatic force (for a neutral surface) nor hydrogen bonds could be implicated.

The next step was dedicated to study the role of forces between molecules, and their competition with molecule-surface VdW terms. We placed a first molecule on the surface at the previously identified minimum free-energy site, while a second molecule was placed at random in a nearby position sufficiently distant from the first one to freely move without directional bias. The $x$ and $y$ coordinates of the center of mass of the second molecule are used as CV. Although both molecules are free to move, the low temperature of $T=1 \mathrm{~K}$ keeps in practice the first molecule always at its initial position, during the whole simulation. The same set of parameters described above were used to perform the MtD simulation.

Figure 3 shows the most favorable geometries of the dimer for the THB and TBB molecules, where the molecular structure is superimposed on an map showing the excess free energy of the second molecule (intermolecular + molecule-surface). In either case, both molecules of the dimer occupy a hollow site. Three arrangements with a similar adsorption energy are found for THB dimers, the one visible in Fig. $3 \mathrm{~b}$ corresponding to the one experimentally observed in a monolayer of THB molecules. ${ }^{11}$ For the TBB dimer a single free energy minimum is obtained, with a rotation of one molecule by $60^{\circ}$ with respect to the other, consistent with the experimental observations. ${ }^{11}$

Figure 4 shows the most favorable geometry of the dimer for THBB (Fig. 4 a,b) and TBBB (Fig. 4 c). As before, the color map in the figure displays only the excess free-energy with respect to the single-molecule value, already shown in the previous figures. For both types of molecules, a free energy minimum is found when two arms from adjacent molecules are parallel to each other. In terms of intermolecular energy, this conformation maximizes the VdW and electrostatic interaction between the molecules. For the THBB molecule a second minimum, with almost the same free energy, is observed when both molecules have the same orientation. For both molecules, it can be noticed that the arms tend to align to the triangular symmetry of the underlying Si:B (111) surface lattice. This effect, coupled with the different degree of overlap between the arms of the 
two molecules, makes for a slight difference in the arrangement of the two dimers on the surface (see Fig. 4).

\section{Condensed-phase Molecular dynamics}

A recent experimental study of the self-assembly of THB and TBB molecules onto the Si:B (111) surface $^{11}$ revealed the formation of either close-packed, or open supramolecular architectures, depending on the molecule termination. For both molecules, it was noticed that the molecular lattice shows a periodic arrangement in which the basic repeat unit consists of the molecular dimer, consistent with the one found above with MtD simulations. In MD, the free-energy driven transformations of one two-dimensional molecular lattice into another can be very complex to probe, due to multiple close-lying potential energy minima, slow single-molecule diffusivity, and presence of multi-molecule concerted displacements (all hardly observable in a standard MD simulation). Therefore, it is customary to define a few initial molecular configurations with different symmetries, some of which close to the ones experimentally observed. Keeping as a basic pattern the molecular dimer issued from the MtD simulations and based on the molecular symmetries observed for the small THB and TBB molecules, we built three tentative molecular lattices, for both THBB and TBBB molecules, as an initial guess.

In the first configuration (see Fig. 5a and Fig. 6a), each arm of a molecule strongly overlaps (in terms of the $\mathrm{VdW}$ spheres) with the arm of a nearby molecule, thus optimizing saturation of the intermolecular $\mathrm{VdW}$ and electrostatic interactions. Note that in the case of Br-terminated molecules, also 3 H-bonds per molecule are formed. This configuration results in a low-density (LD) hexagonal lattice, with the two unit cell vectors of length $a$ at $120^{\circ}$ and two molecules per unit cell with the minimum-energy dimer shape. As it can be seen in Fig. 5a and Fig. 6a, such a lattice contains large hexagonal pores, spaced by the same distance $a$.

In the second configuration (Fig. 5b and Fig. 6b), two out of three arms in each molecule inter-

act with the corresponding arms of two nearby molecules, giving a semi-compact (Mix) hexagonal lattice, this time containing 6 molecules per unit cell of size $a^{\prime}$. In this lattice one can identify three 
sets of pores: a large one with area $A$ (different for each molecule), a medium size one, elongated, with area $\approx 0.6 A$, and a small one surrounding an isolated adatom, with area $\approx 0.4 A$. All the three sets are arranged on a different hexagonal lattice, with the same spacing $a^{\prime}$, but shifted with respect to each other.

In the third one (Fig. 5c and Fig. 6c), two of the arms interact with neighboring molecules, albeit to a different degree of overlap. In this case, two molecules per unit cell are arranged at the vertices of a slightly distorted rectangular lattice, with lattice vectors $a$ and $b$ at nearly $90^{\circ}$, a higher molecular density (HD), and practically no surface porosity.

Notably, to build the LD and HD periodic lattices, the molecules need to rotate by about $19^{\circ}$ compared to the MtD configuration of Fig. 4. This rotation causes a slight overlap of the arms of the molecule with the rows of adatoms. For the "Mix" lattice such a rotation is not necessary. For both molecules in each of the three lattices, we built systems containing between 24 and 28 molecules each, according to the density, arranged on top of 12x12 (for THBB) and 14x14 (for TBBB) units of the Si:B (111) reconstructed surface. The thickness of the Si slab in the perpendicular direction was of three (111) planes, i.e. $9 \AA$, for a total of 2592-3528 Si and 144-196 B atoms for the $12 \times 12$ and 14x14 surfaces respectively. Ample void slabs were added above and below in the z direction, to avoid image self-interaction. All the MD simulations were carried out with the velocity-Verlet integrator, and a time step of $1 \mathrm{fs}$.

\section{Results for the THBB molecule}

The optimized values of the lattice parameters for the three molecular lattices built from THBB molecules are summarized in Table 1 . After MD equilibration at $T=50 \mathrm{~K}$ with canonical ensemble constraints (constant number of molecules $N$, volume $V$ and temperature $T$ ), the structures are quenched down to zero temperature, to compare the initial and final configuration of each of the three lattices (Fig. 5). For the THBB molecule, only the "Mix" lattice is stable and retains

the initial symmetry, albeit with some slight relative adjustment of the molecules. Conversely, in the LD lattice molecules start turning and bending some of the arms, departing from the $\mathrm{C}_{3 v}$ 
planar symmetry. The lattice is strained and partly disordered, becoming incommensurable with the underlying Si:B surface. The disordering is even worse for the HD lattice, in which molecules completely disrupt the symmetry, tilt and rotate, and start overlapping with each other.

From the average values of VdW intermolecular (MM) and molecule-surface (MS) energy contributions reported in Table 2, it is seen that the "Mix" lattice has a MS energy almost 10\% lower than the one of the LD and HD lattices, in great part coming from the better commensurability of the "Mix" lattice with the surface. The MM energies, in comparison, are much closer to each other within the error bars. Therefore, it appears that the increased stability in this case originates from a better match of the "Mix" lattice with the preferred surface adsorption sites.

\section{Results for the TBBB molecule}

In contrast to the THBB molecule, the Br-terminated (TBBB) molecule enables the formation of a hydrogen bond between each terminal $\mathrm{Br}$ atom and one $\mathrm{H}$ from a nearby molecule. This interaction, which may be important in the final arrangement of the molecular lattice, has been explicitly taken into account in the MD simulation by adding a $\mathrm{H}$-bond interaction between selected pairs of atoms. ${ }^{41}$ In practice, we allow the formation of a unique $\mathrm{Br}-\mathrm{H}$ bond per arm, between two different molecules, by looking among all the possible $\mathrm{H}$ surrounding the $\mathrm{Br}$ atom, and picking the one which is closest to the generic properties of a H-bond. ${ }^{41,42}$ Since values of the strength of the $\mathrm{Br}-\mathrm{H}$-bond do not seem to be available in the literature, ${ }^{43,44}$ we assigned it an approximate value of $1 \mathrm{kcal} / \mathrm{mol}$, which seems a reasonable value compared to other similar molecular configurations. (As it will be apparent later, the exact absolute value does not seem to affect the results, since $\mathrm{H}$ bonds have a comparable role in all the configurations.) In this way, three $\mathrm{H}$-bonds per molecule are added, in each of the three starting lattices (see Fig. 6 a,b,c). For the TBBB molecule, whose lattice parameters are summarized in Table 4, the initial molecular arrangement is slightly less dense in comparison with the THBB molecule. Initial and final configurations after MD equilibration by constant-(NVT) at $T=50 \mathrm{~K}$, followed by quenching at $T=0 \mathrm{~K}$, are shown in Figure 6 . In this case only the LD lattice is stable, while disordering with internal tilting and rotation of individual 
molecules is observed for both the "Mix" and HD lattices. In the stable LD lattice the molecules tend to tighten their relative distances, causing a loss of the commensurability with the underlying $\mathrm{Si}: \mathrm{B}(111)$ lattice.

As for the previous case, Table 3 summarizes the MM vs. MS VdW energies for the TBBB molecule. It can be seen that, contrary to the THBB, the variation of MS interaction across the three lattices is smaller here, indicating a relative irrelevance of the surface in stabilizing the lattice. On the other hand, the MM VdW energy for the LD lattice is in this case definitely more favorable than the corresponding values for the HD and "Mix" lattice.

It may be thought that for the TBBB molecule, the extra H-bonds could be the essential feature

inducing the intermolecular interaction predominance. However, when looking at the H-bond contributions for the three lattices, we find an average of $-1.5 \mathrm{kcal} / \mathrm{mol}$ per molecule for the LD and "Mix" lattices, and $-1.9 \mathrm{kcal} / \mathrm{mol}$ per molecule for the HD lattice. While the H-bond energy adds almost $15-20 \%$ to the MM energy in each lattice, its relative value is practically the same for the three lattices, therefore it could not be responsible for the extra stability of the LD. It is clearly the difference in VdW interaction that increases the MM energy, up to the point of stabilizing the LD lattice.

It is also worth noting that the contribution of electrostatic interactions to the MM energy, although large in absolute value, is nearly identical in both molecules for all the lattices (between 67.3 and $67.5 \mathrm{kcal} / \mathrm{mol}$ for the THBB, and between -71.9 and -72.4 for the TBBB), therefore it cannot possibly contribute any significant difference in the stability of one symmetry with respect to another. In fact, it is seen that nearly all of the electrostatic energy comes from interactions within each molecule (and, therefore, is independent on the lattice symmetry), while the intermolecular contribution is practically negligible compared to the VdW contribution.

\section{Comparison with STM experiments}

Finally, to verify the predictive value and the accuracy of the MtD+MD simulations, we compared the above results to the experiments on self-assembled supramolecular structures. Single monolay- 
ers consisting of either the THBB or TBBB molecules were deposited on the Si:B (111) surface, as described above in the Materials and Methods section. For both types of molecule, a unique arrangement was always observed by scanning tunneling microscopy (STM).

In the case of the THBB molecule, each molecule is well-resolved in the STM image of Figure 7(a). The molecules have a clear ternary symmetry, where two arms appear brighter than the third one. In contrast to their smaller THB counterpart, ${ }^{11}$ their self-assembly does not lead to a closepacked arrangement. Indeed, the molecular lattice contains regular pores, which define a unit cell comprising six molecules arranged in a compact structure. Superimposing the "Mix" molecular lattice to the STM image reveals a very good agreement between the theoretical prediction and the experimental result. It is worth noting that simply restricting our initial guess to the closed-packed structure, as deduced from the lattice of the THB molecules, would not have lead the MD simulations to correctly identify the most stable configuration observed here. The systematic comparison of the competing interactions between both molecules suggests the increasing importance of the molecule-surface interaction, as the size of the molecular backbone increases.

As to the TBBB molecules, Figure 7(b) shows a STM image of the molecular structure after adsorption on the $\mathrm{Si}: \mathrm{B}$ (111) surface. It is seen that the three arms of each molecule do not show an homogeneous contrast over the whole molecule. As a result, the assignment of the molecular backbone arrangements in this case requires more attention. However, also in this case the selfassembly clearly leads to a porous molecular lattice, where each pore is defined by the end-group of six molecules, similarly to the molecular lattice experimentally observed for the TBB molecules. ${ }^{11}$ Each molecule thus connects three adjacent pores, consistently with the preferred LD structure found in MD (nicely superimposed to the experimental image in Fig. 7(b)). Remarkably, the slight shift of the center of mass of each molecule with respect to the hollow site lattice, accounts for the existence of pores with different geometries. The MD simulation allows to reconcile the observation of the dim Si adatoms in the pores, with the size and geometry of the pores. 


\section{Conclusions}

By using a combination of atomic-scale simulation methods, we successfully predict and demonstrate the stability of two-dimensional ordered surface lattices, for two similar planar molecules deposited on a passivated $\mathrm{Si}: \mathrm{B}(111)$ surface. The two molecules differ in their terminations, whence the group at the end of each of the three biphenyl arms attached to a central carbon ring can be either a $\mathrm{H}$ or a $\mathrm{Br}$, the latter being able to form extra hydrogen bonds, compared to the former. We use an improved empirical description of molecule-surface Van der Waals forces, based on a novel parametrization from ab-initio TD-DFT calculations providing the correct asymptotic nearfield radial behavior. The predicted equilibrium molecular lattices compare extremely well with the experiments, in which monolayers of the same molecules are deposited on the $\mathrm{Si}: \mathrm{B}$ surface, and imaged by scanning-tunneling microscopy (STM). We find that the H-terminated molecule optimizes the "Mix" lattice by favouring the VdW interaction with the surface, since the calculated molecule-surface VdW energy is almost $10 \%$ larger in the stable configuration, compared to any other configuration studied. A different situation is observed in the case of the Br-terminated molecule. The calculated VdW energy for the molecule-surface interaction is here the smallest for the most stable lattice. However, the large gain in intermolecular interaction is the key in stabilizing the LD structure. The eventual formation of hydrogen bonds does not seem to quantitatively affect the results, in that the competition between surface and molecular VdW forces appears to be also in this case the most important determinant for the preferred structures.

These results shows how predictions of self-assembled structures at semiconductor surfaces can be highly non-trivial, depending on a subtle balance between non covalent forces, which are however integrated over large contact lengths and areas, thereby producing large, competing contributions to the total energy. Computer simulations provide deep insight into the interaction strength driving the self-assembly, and enable to assess the subtle interplay of the Van der Waals interactions, compared to chemically-specific interactions such as hydrogen bonding. In addition, it is worth noting that such simulations also offer a great support to the analysis of experimental data. For example, STM images probe the local electron density of states and, as a result, the images 
might not always correctly reflect the actual molecular structure. Simulation tools which allow to compare the geometry of competing supramolecular architectures against the one observed by STM, can be particularly useful to identify the actual molecular arrangements, and to correlate the STM features with the corresponding molecular orbitals and bonding sites. 


\section{References}

(1) Mann, S. Nat. Mater. 2009, 8, 781.

(2) Sihgha, A.; Gibertini, M.; Karmakar, B.; Yuan, S.; Polini, M.; Vignale, G.; Katnelson, M. I.; Pinczuk, A.; Pfeiffer, L. N.; West, K. W.; Pellegrini, V. Science 2011, 332, 1176.

(3) Gomes, K. K.; Mar, W.; Ko, W.; Guinea, F.; Manoharan, H. C. Nature 2012, 483, 306.

(4) Liu, Z.; Wang, Z.-F.; Mei, J.-W.; Wu, Y.-S.; Liu, F. Phys. Rev. Lett. 2013, 110, 106804.

(5) Bartels, L. Nature Chem. 2010, 2, 87.

(6) Stepanow, S.; Lingenfelder, M.; Dmitriev, A.; Spillmann, H.; Delvigne, E.; Lin, N.; Deng, X. B.; Cai, C. Z.; Barth, J. V.; Kern, K. Nat. Mater. 2004, 3, 229.

(7) Lehn, J.-M. Supramolecular Chemistry: Concepts and Perspectives, VCH,Weinheim ed.; 1995.

(8) Makoudi, Y.; Palmino, F.; Arab, M.; Duverger, E.; Chérioux, F. J. Am. Chem. Soc. 2008, 130, 6670.

(9) Winfree, E.; Liu, F.; Wenzler, L. A.; Seeman, N. C. Nature 1998, 394, 539.

(10) Bocquet, F.; Nony, S.; Mannsfeld, C. B.; Oison, V.; Pawlak, R.; Porte, L.; Loppacher, C. H. Phys. Rev. Lett. 2012, 108, 206103.

(11) Makoudi, Y.; Baris, B.; Jeannoutot, J.; Palmino, F.; Grandidier, B.; Chérioux, F. Chem. Phys. Chem. 2013, 14, 900.

(12) Baris, B.; Luzet, V.; Duverger, E.; Sonnet, P.; Palmino, F.; Chérioux, F. Angew. Chem. Int. Ed. 2011, 50, 4094.

(13) Baris, B.; Jeannoutot, J.; Luzet, V.; Palmino, F.; Rochefort, A.; Chérioux, F. ACS Nano 2012, 6,6905 . 
(14) Wagner, S. R.; Lunt, R. R.; Zhang, P. Phys. Rev. Lett. 2013, 110, 086107.

(15) Krishnan, M.; Balasubramanian, S.; Clarke, S. J. Chem. Phys. 2003, 118, 5082.

(16) Müller, T.; Werblowsky, T. L.; Florio, G. M.; Berne, B. J.; Flynn, G. W. PNAS 2005, 102, 5315.

(17) Kannappan, K.; Werblowsky, T. L.; Rim, K. T.; Berne, B. J.; Flynn, G. W. J. Phys. Chem. B 2007, 111, 6634.

(18) Ciesielski, A.; Szabelski, P. J.; Rzysko, W.; Cadeddu, A.; Cook, T. R.; Stang, P. J.; Samori, P. J. Am. Chem. Soc. 2013, 135, 6942.

(19) Cleri, F. Phys. Rev. B 2009, 80, 235406.

(20) Champness, N. R. Nature Nano. 2007, 2, 671.

(21) Mendez, J.; Lopez, M. F.; Martin-Gago, J. A. Chem. Soc. Rev. 2011, 40, 4578.

(22) Lukas, S.; Witte, G.; Wöll, C. PRL 2002, 88, 028301.

(23) Fernandez-Torrente, I.; Monturet, S.; Franke, K. J.; Fraxedas, J.; Lorente, N.; Pascual, J. PRL 2007, 99, 176103.

(24) Silien, C.; Räisänen, M. T.; Buck, M. Small 2010, 6, 391.

(25) Breitwieser, R.; Marsault, M.; Repain, V.; Lagoute, J.; Chacon, C.; Girard, Y.; Rousset, S.; Qin, Z.; Battaglini, N.; Zrig, S.; Lang, P. J. Chem. Phys. 2013, 139, 204703.

(26) Frisch, M. J.; Trucks, G. W.; Schlegel, H. B.; Scuseria, G. E.; Robb, M. A.; Cheeseman, J. R.; Montgomery, J. A., Jr.; Vreven, T.; Kudin, K. N.; Burant, J. C.; et al., Gaussian 03, Revision C.03. Gaussian, Inc., Wallingford, CT, 2004.

(27) Todorov, I. T.; Smith, W.; Trachenko, K.; Dove, M. T. J. Mater. Chem. 2006, 16, 1911. 
(28) Bonomi, M.; Branduardi, D.; Bussi, G.; Camilloni, C.; Provasi, D.; Raiteri, P.; Donadio, D.; Marinelli, F.; Pietrucci, F.; Broglia, R. A.; Parrinello, M. Comp. Phys. Comm. 2009, 180, 1961.

(29) Laio, A.; Parrinello, M. Proc. Natl. Acad. Sci. USA. 2002, 99, 12562.

(30) Stillinger, F.; Weber, T. A. Phys. Rev. B 1985, 31, 5262.

(31) Rasband, P. B.; Clancy, P.; Roberts, B. W. J. Appl. Phys. 1998, 84, 2471.

(32) Allinger, N. L.; Yuh, Y. H.; Lii, J. H. J. Am. Chem. Soc. 1989, 111, 8551-8576.

(33) Allinger, N. L.; Li, F.; Yan, L. J. Comput. Chem. 1990, 11, 848.

(34) Zaremba, E.; Kohn, W. Phys. Rev. B 1976, 13, 2270.

(35) Oliveira, M. J. T.; Botti, S.; Marques, M. A. L. Phys. Chem. Chem. Phys 2011, 13, 15055.

(36) Lu, J.; Tao, Y.; D’Iorio, M.; Li, Y.; Ding, J.; Day, M. Macromol. 2004, 37, 2442.

(37) Chérioux, F.; Guyard, L.; Audebert, P. Chem. Commun. 1998, 2225.

(38) Chérioux, F.; Guyard, L. Adv. Funct. Mater 2001, 11, 305.

(39) Bedrossian, P.; Robert, M. D.; Mortensen, K.; Chen, D. M.; Golovchenko, J. A.; Vanderbilt, D. Phys. Rev. Lett. 1989, 63, 1257.

(40) Melis, C.; Colombo, L.; Mattoni, A. J. Phys. Chem. C 2011, 115, 18208.

(41) Lii, J.-H.; Allinger, N. L. J. Comp. Chem. 1998, 19, 1001.

(42) Mayo, S. L.; Olafson, B. D.; III, W. A. G. J. Phys. Chem. 1990, 94, 8897.

(43) Lieberman, H. F.; Davey, R. J.; Newsham, D. M. T. Chem. Mater. 2000, 12, 490.

(44) Navon, O.; Bernstein, J.; Khodorkovsky, V. Angew. Chem. Int. Ed. Engl. 1997, 36, 601. 


\section{Tables}

Table 1: Lattice parameters of the 2D unit cell, and calculated density for the LD, Mix and HD lattice of the THBB molecule.

\begin{tabular}{lcc}
\hline \hline & Parameter $(\AA)$ & Density $\left(10^{3} \mathrm{~mol} / \AA^{2}\right)$ \\
\hline LD & $a=23.4$ & 0.40 \\
Mix & $a=40.4$ & 0.42 \\
HD & $a=15.5, b=26.1$ & 0.50
\end{tabular}

Table 2: Average values of Van der Waals contributions to intermolecular, and molecule-surface energy for the 2D lattices formed by the THBB molecule.

\begin{tabular}{lcc}
\hline \hline & VdW MM (kcal/mol) & VdW MS (kcal/mol) \\
\hline LD & $-9.1 \pm 1.3$ & $-33.8 \pm 2.2$ \\
Mix & $-8.7 \pm 1.4$ & $-36.9 \pm 4.1$ \\
HD & $-9.9 \pm 1.0$ & $-33.0 \pm 4.3$
\end{tabular}

Table 3: Average values of Van der Waals contributions to intermolecular, and molecule-surface energy for the 2D lattices formed by the TBBB molecule.

\begin{tabular}{ccc}
\hline \hline & VdW MM (kcal/mol) & VdW MS (kcal/mol) \\
\hline LD & $-9.9 \pm 0.4$ & $-41.2 \pm 0.5$ \\
Mix & $-7.5 \pm 2.0$ & $-43.5 \pm 2.0$ \\
HD & $-6.1 \pm 1.4$ & $-42.7 \pm 1.3$
\end{tabular}


Table 4: Lattice parameters of the 2D unit cell, and calculated density for the LD, Mix and HD lattice of the TBBB molecule.

\begin{tabular}{lcc}
\hline \hline & Parameter $(\AA)$ & Density $\left(10^{3} \mathrm{~mol} / \AA^{2}\right)$ \\
\hline LD & $a=26.7$ & 0.33 \\
Mix & $a=44.4$ & 0.32 \\
HD & $a=17.5, b=31.0$ & 0.37
\end{tabular}




\section{Captions}

figure 1 Ball and stick representation of the 1,3,5-tris(phenyl)benzene (THB upper left), 1,3,5tris(4'-biphenyl)benzene (THBB lower left), 1,3,5-tris(4'-bromophenyl)benzene (TBB upper right) and 1,3,5-tris(4"'-bromobiphenyl)benzene (TBBB lower right) molecule with cyan for carbon, white for hydrogen and green for brome atoms.

figure 2 Mapping of the free energy profile estimated for the THB (a), TBB (b), THBB (c) and TBBB (d) molecules on the Si:B (111) surface. Black dots represent silicon adatoms. Energy-scale are in $\mathrm{kcal} / \mathrm{mol}$.

figure 3 (a),(b),(c) Mapping of the free energy profile estimated for a dimer of THB on Si:B (111) surface. Stick representation of the molecules are superimposed on the profile to show the dimers configurations. (d) Mapping of the free energy profile estimated for a dimer of TBB on the Si:B (111) surface. Stick representation of the dimers configuration are superimposed to the profile. Black dots represent silicon adatoms. Energy-scale are in $\mathrm{kcal} / \mathrm{mol}$.

figure 4 (a),(b) Mapping of the free energy profile estimated for THBB dimers on Si:B (111) surface. Stick representation of the dimers configuration are superimposed on the profile. (c) Mapping of the free energy profile for TBBB dimers on the Si:B (111) surface. Configuration of the molecules are represented by stick and superimposed on the profile. Black dots represent silicon adatoms. Energy-scale are in $\mathrm{kcal} / \mathrm{mol}$.

figure 5 Left: initial configuration of the (a) LD, (b) Mix and (c) HD molecular lattice for THBB. Right: final configuration of the (a) LD, (b) Mix and (c) HD molecular lattice, after equilibration at $T=50 \mathrm{~K}$ by $\mathrm{MD}$ at constant-(NVT). Molecules are represented by cyan sticks for $\mathrm{C}$ atoms and white sticks for $\mathrm{H}$ atoms. Black dots represent the underlying $\mathrm{Si}$ adatoms with a $\sqrt{3} \times \sqrt{3}$ periodicity. Geometric shapes on the left images represent the unit cell of the molecular lattice.

figure 6 Left: initial configuration of the (a) LD, (b) Mix and (c) HD molecular lattice for TBBB. Right: final configuration of the (a) LD, (b) Mix and (c) HD molecular lattice, after equilibration at $T=50 \mathrm{~K}$ by $\mathrm{MD}$ at constant-(NVT). Molecules are represented by cyan sticks for C 
atoms, white sticks for $\mathrm{H}$ atoms and green stick for $\mathrm{Br}$ atoms. Geometric shapes on the left images represent the unit cell of the molecular lattice.

figure 5 Comparison between experimental STM image (background) and simulation (foreground) for the THBB (a) and the TBBB (b) supramolecular lattices. 
Figures

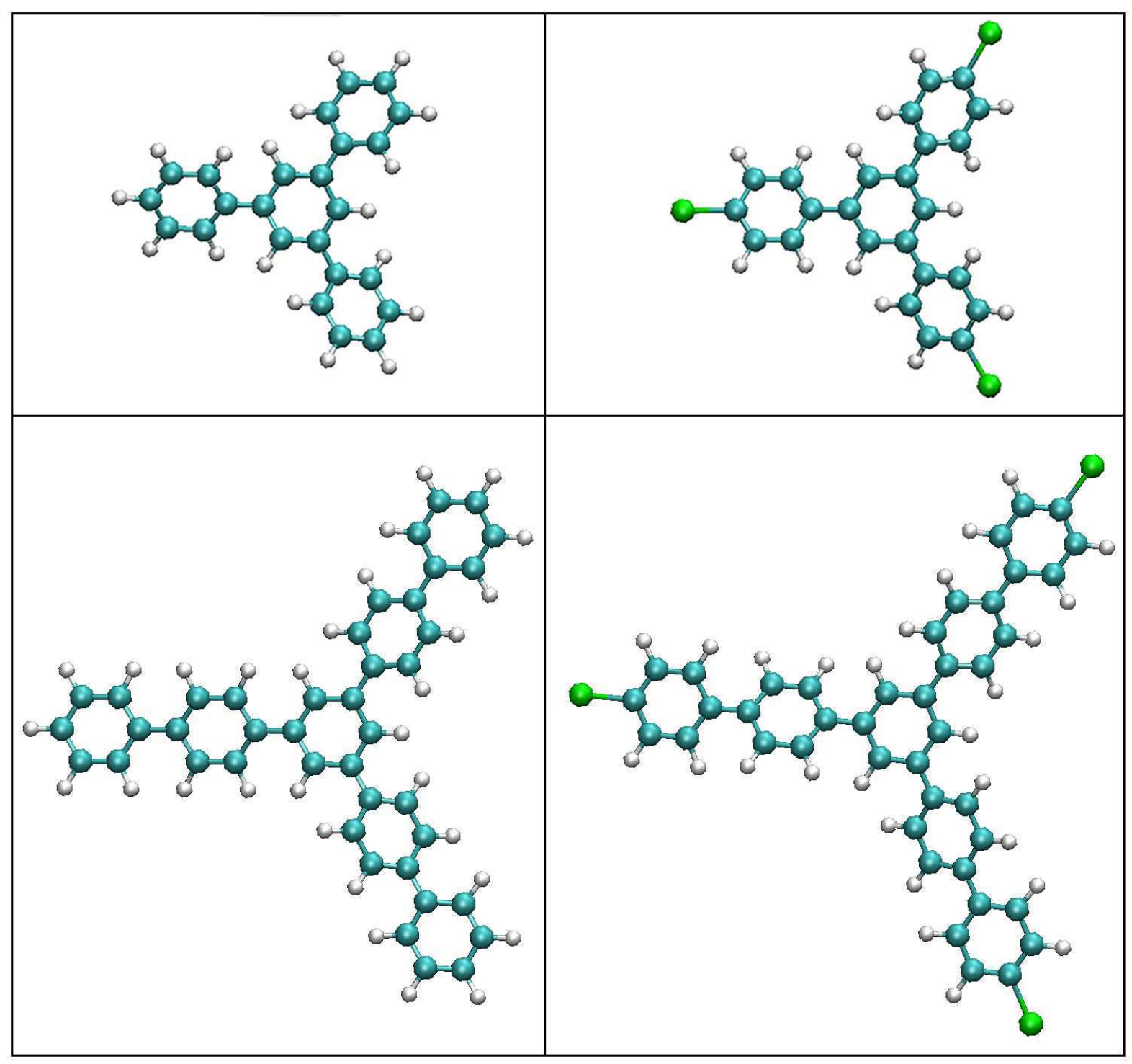

Figure 1 

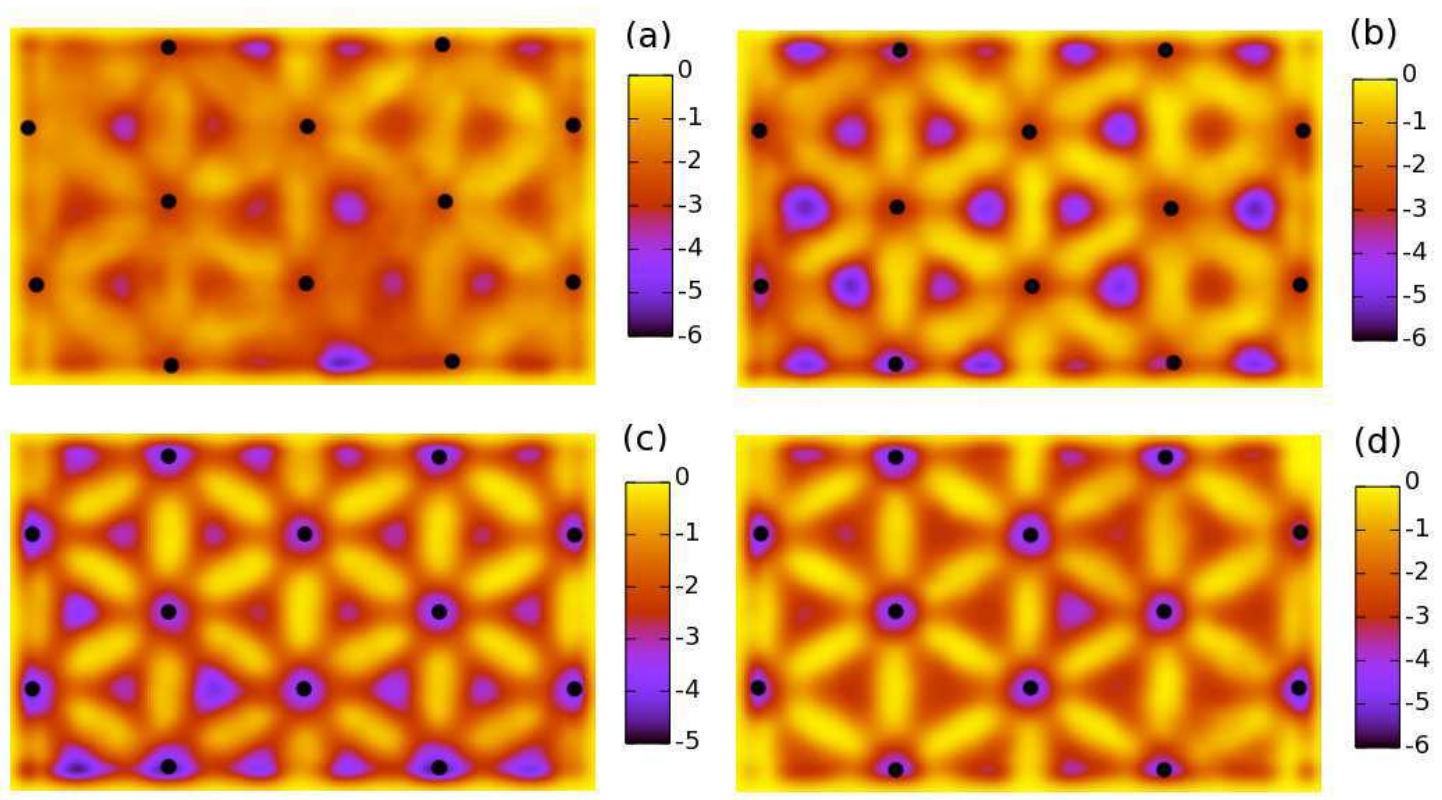

Figure 2 


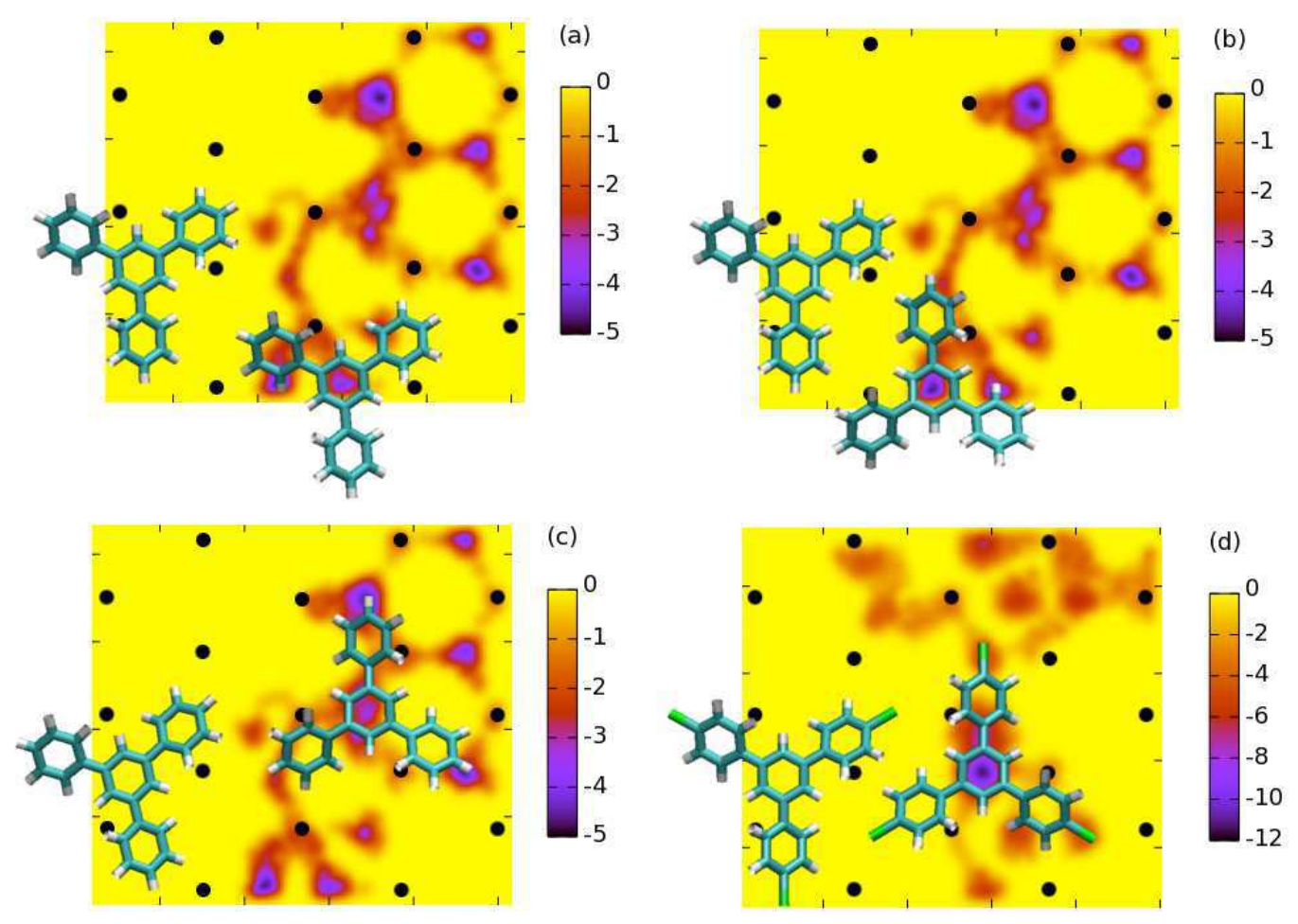

Figure 3 

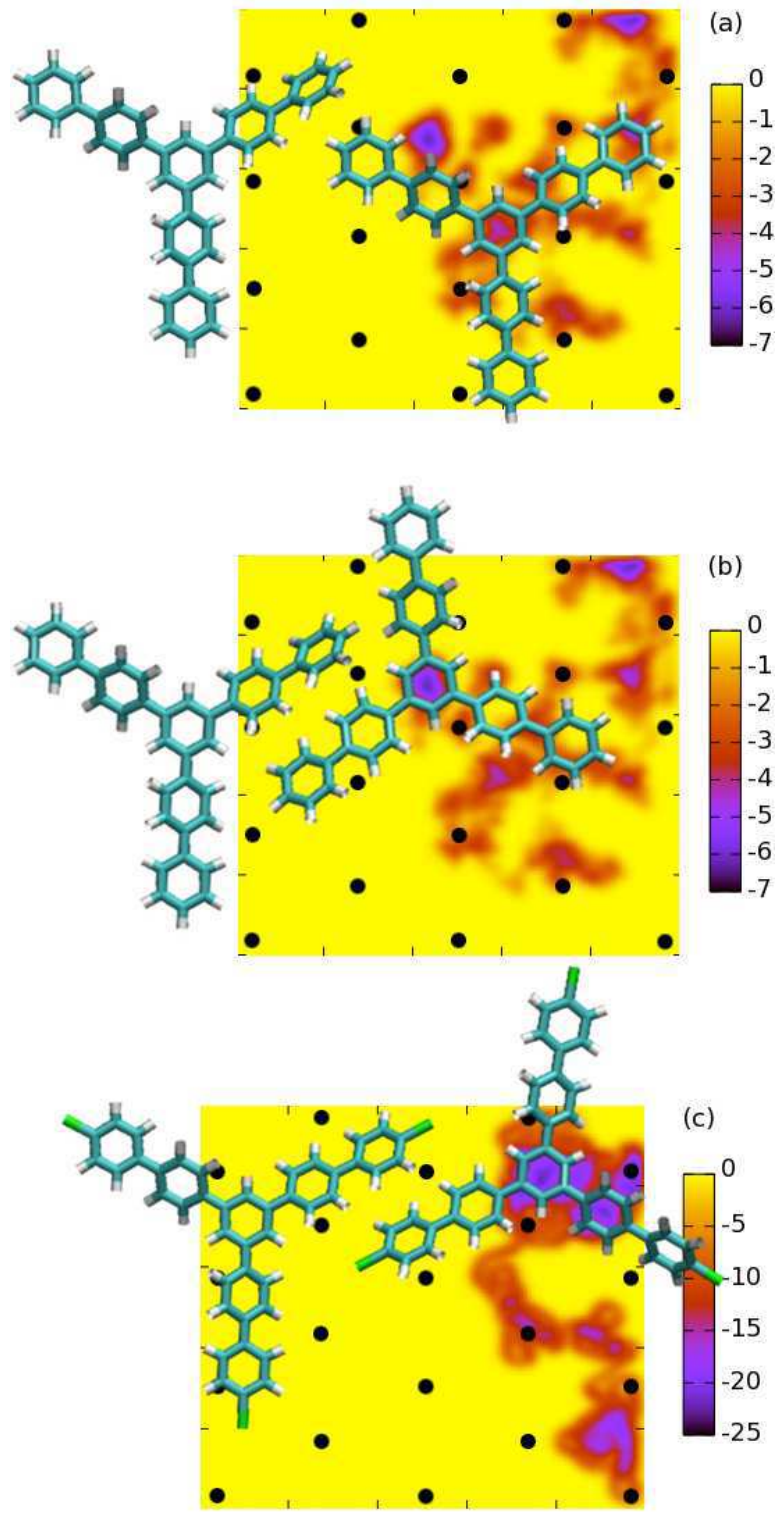

Figure 4 


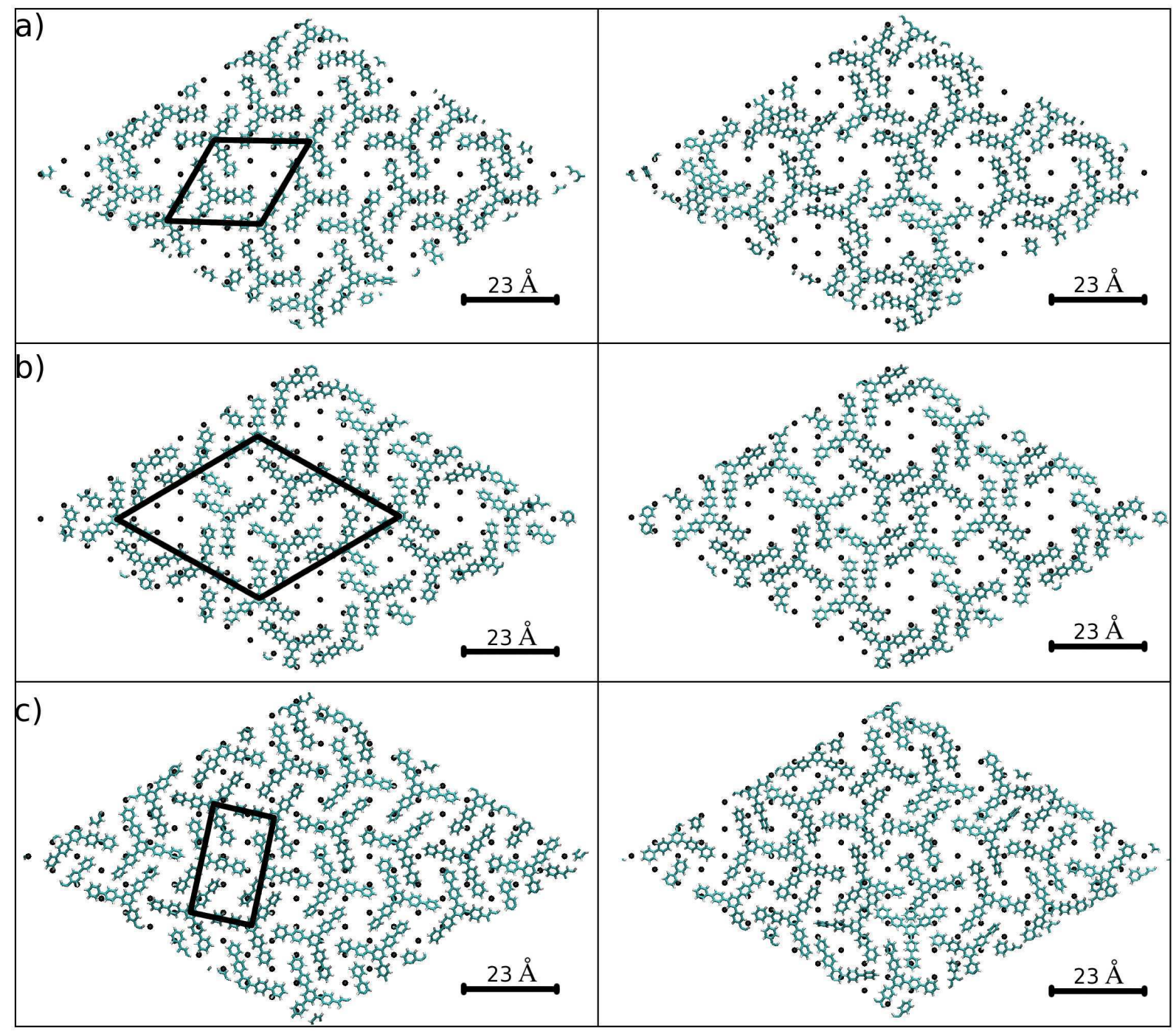

Figure 5 


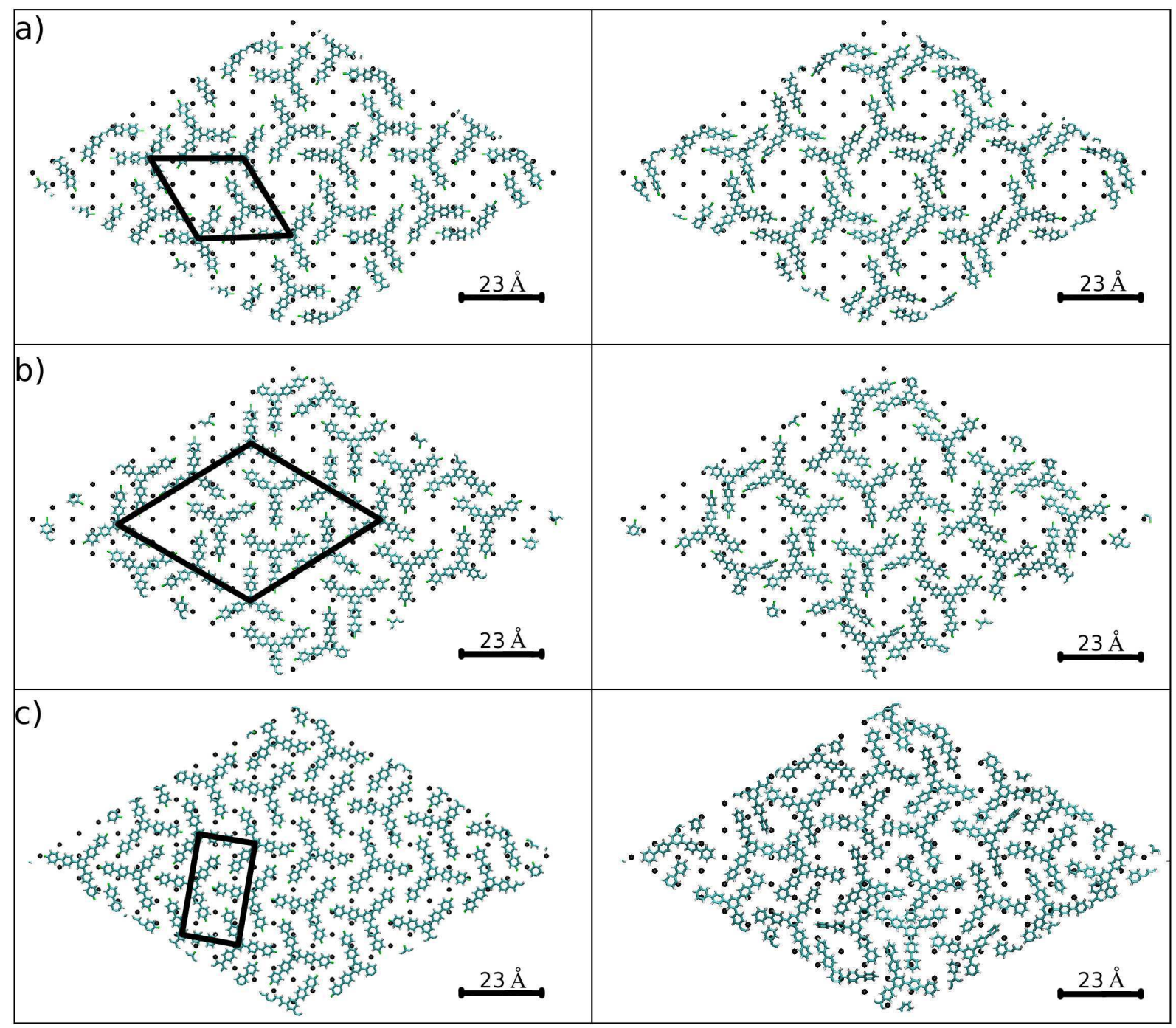

Figure 6 


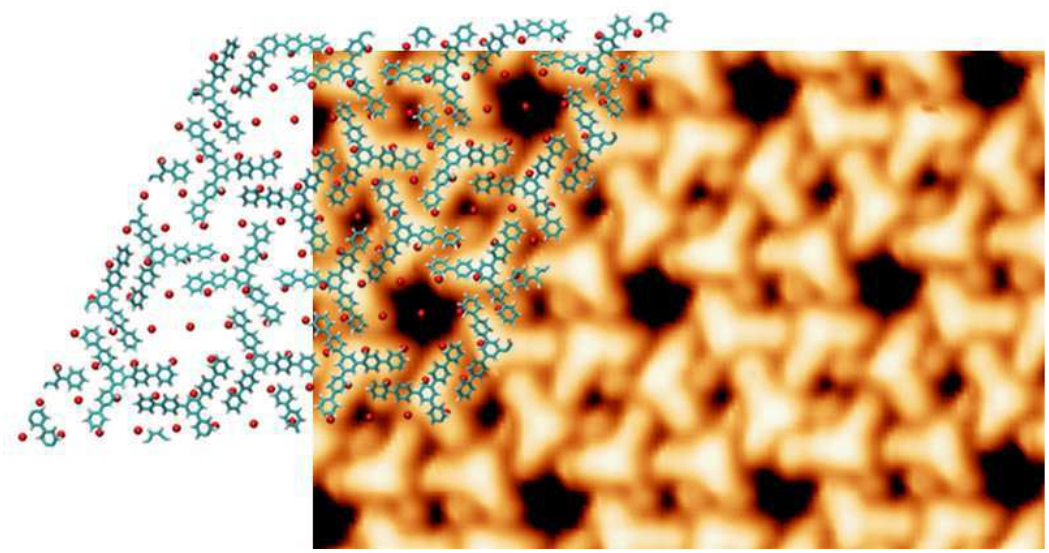

(a)

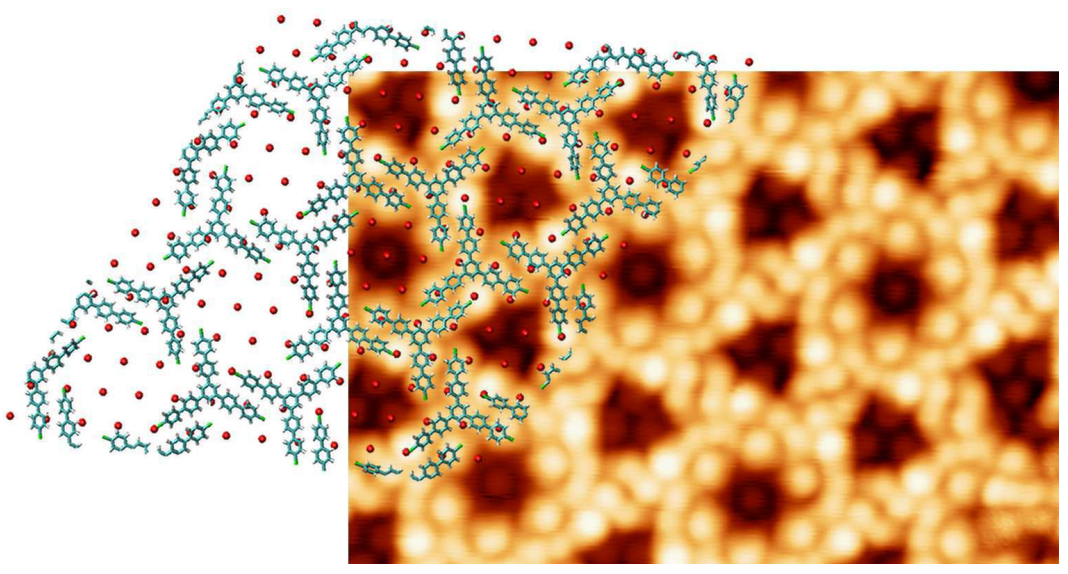

(b)

Figure 7 